\title{
Total Pancreactectomy with Islet Autotransplant Failure: Now What?
}

\author{
Ty B. Dunn ${ }^{1}$ - Varvara A. Kirchner ${ }^{1} \cdot$ K. Louise Berry ${ }^{1} \cdot$ Melena D. Bellin $^{2}$. \\ David E. R. Sutherland ${ }^{1}$ - Timothy L. Pruett ${ }^{1}$
}

Published online: 7 April 2015

(C) Springer International Publishing AG 2015

\begin{abstract}
Total pancreatectomy with islet cell autotransplant (TPIAT) is a definitive therapeutic approach to pain management for patients with chronic pancreatitis that is nonresponsive to maximum medical management. Total pancreatectomy (TP) results in brittle diabetes unless islet cell autotransplant (IAT) is concomitantly performed. Even with IAT, about $30 \%$ of patients will require long-term basal-bolus insulin therapy due to significant endocrine insufficiency. The focus of this review is on the utility of whole organ pancreas transplantation toward improving quality of life, and decreasing the risk of mortality and overall cost of care for the subset of patients who experience endocrine and exocrine deficiency after pancreatic resection.
\end{abstract}

Keywords Pancreas transplant · Total pancreatectomy · Brittle diabetes $\cdot$ Cost $\cdot$ Quality of life $\cdot$ Survival

\section{Introduction}

Total pancreatectomy with islet cell autotransplant (TPIAT), first performed in 1977, has emerged in recent years as an increasingly recognized treatment option for intractable chronic and acute relapsing pancreatitis refractory to maximal medical therapy $[1 \bullet, 2 \bullet \cdot 3 \bullet \bullet, 4 \bullet \cdot$. All patients undergoing partial or

This article is part of the Topical Collection on Pancreas Transplantation

Ty B. Dunn

dunn0017@umn.edu

1 Department of Surgery, University of Minnesota, 420 Delaware St. SE, MMC 195, Minneapolis, MN 55455, USA

2 Department of Pediatrics, University of Minnesota, Minneapolis, MN, USA total pancreatic resectional procedures (with or without islet cell transplant) are at significant long-term risk for both endocrine and exocrine insufficiency in varying degrees [5•]. About 60 $70 \%$ of patients at 3 years after TPIAT either have full or partial endocrine function with a hemoglobin A1c $<7.0[2 \bullet \bullet, 3 \bullet \bullet]$. For the remaining patients who require typical basal-bolus insulin therapy due to insufficient islet cell mass, diabetes is usually manageable with current day therapies such as insulin pumps and glucose sensors, unless the islet cell autograft cannot maintain c-peptide positivity. Similarly, most patients do well on pancreatic enzyme replacement therapy (PERT), with control of steatorrhea and absence of serious malnutrition/vitamin deficiency issues. Patients who experience significant exocrine issues experience variable absorption of carbohydrates and are at highest risk for the development of increasingly poor blood glucose control over time [6••], with subsequent hypoglycemic unawareness and secondary complications of diabetes. These are also the patients at highest risk for nutritional deficiencies. The focus of this review is on the utility of whole organ pancreas transplant alone (PTA) toward improving the quality of life, risk of mortality, and overall cost of care for the subset of patients who develop uncontrolled endocrine or exocrine failure after pancreatic resection.

\section{Indications}

Importantly, indications for TPIAT and pancreas transplant are distinctly different. Pancreatic resection (total or partial) is offered to remove the source of intractable pain and root cause of futile medical management and dysfunctional lifestyle. These issues result from recurrent emergency department and inpatient visits for pain and dehydration as well as repeated endoscopic therapies (ERCP and feeding tubes) with progressive and significant disability due to loss of social functioning (inability to attend school, 
maintain employment, and perform family roles) and subsequent development of psychological distress (depression and anxiety). The islet cell autotransplant (IAT) portion of the procedure simply relocates the patient's own islet cells in order to preserve endocrine function, therefore no immunosuppression is required.

In contrast, PTA is offered to replace missing function for diabetics (Type 1 and some Type 2) who, despite maximal medical management, experience uncontrolled diabetes and hypoglycemic unawareness. The native pancreas is not removed, and all patients require immunosuppression to prevent rejection of the allograft. There has been a recent increased awareness of patients with both endocrine and exocrine deficiencies due to pancreatic disease (Type $3 \mathrm{c}$ diabetes). Prevalence of Type $3 \mathrm{c}$ diabetes is $5-10 \%$ in Western diabetics, and as high as 15$20 \%$ in Southeast Asia. Causes of this interplay of endocrine and exocrine deficiency remain controversial, with proposed mechanisms including: dysregulation of exocrine secretion due to diabetic neuropathy, atrophy of exocrine tissue because of lack of local trophic effect of insulin, autoimmune damage to both beta-cell and exocrine tissue antigens, and genetic mutations [7••]. This form of diabetes is associated with hormonal abnormalities that are discrete from Type 1 and Type 2 diabetes, which may arise with or without pancreatic resection $[8,9]$.

Total pancreatectomy (TP) without IAT results in an extreme manifestation of pancreatic failure (endocrine and exocrine), with reports of $15-75 \%$ experiencing diabetes control problems [10]. It has been reported that up to $20 \%$ of patients develop secondary diabetic complications after TPIAT with IAT failure [11]. The combination of severe endocrine with exocrine pancreatic failure has been the impetus for selective application of PTA in Type $3 \mathrm{c}$ diabetics [10-12]. Only $0.1 \%$ of all pancreas transplants reported to the International Pancreas Transplant Registry have been for this indication [13]. The true incidence and clinical significance of severe exocrine insufficiency is not known and may be significantly exacerbated by intestinal dysmotility. Exocrine insufficiency after TPIAT has not been well studied due to the overwhelming interest in endocrine outcomes. Functional paracrine/neural elements that are provided though pancreaticoduodenal transplant may provide a more normal physiology of exocrine responsiveness. This aspect of gut physiology is ripe for study.

Despite the disparate indications for TPIAT and PTA, there are significant commonalities: quality of life, quantity of life, and cost containment. These principles are the foundation of modern organ transplantation. Therefore, our center offers PTA whenever endocrine or exocrine control is not achieved with intensive specialty medical care.

\section{Quality of Life}

TPIAT versus TP alone (without IAT) has been associated with a statistically significant increase in the ability to return to work, which illustrates the functional burden of brittle diabetes [14••]. Pancreatectomy for chronic pancreatitis results in good pain relief in most patients, with the majority of patients being able to completely wean off narcotics. Even when not able to discontinue narcotics completely, overall use is substantially lower and functional status is much higher $[2 \bullet \bullet, 4 \bullet \bullet, 14 \bullet \cdot]$ Importantly, in addition to the management of chronic pain, patients with chronic pancreatitis face ongoing loss of endocrine function of their native pancreas secondary to chronic inflammation and fibrosis. This eventually leads to insulin (as well as exocrine) dependence and a need for pancreas transplant. Timely TPIAT, though not without potentially significant complications, gives a chance of pain-free survival and insulin independence.

Once insulin independent, the IAT graft function is quite durable as evidenced by emerging mid- and long-term data

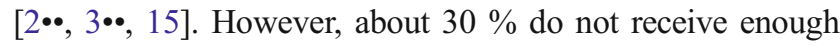
islet mass to remain free of a typical basal-bolus insulin regimen. After intraportal IAT, there is defective glucagon secretion during hypoglycemia that can result in unopposed hypoglycemia that can make serum glucose very difficult to manage $[16 \bullet \bullet$. This sets the stage for oscillating glucose levels, further loss of islet mass, and progression to hypoglycemic unawareness, which is a major contributor to the development of secondary complications of diabetes.

Studies focusing on quality of life metrics have consistently reported significant improvements in both physical

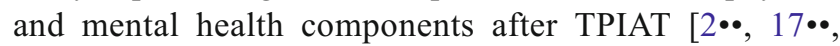
$18 \bullet \bullet$. Likewise, Gross et al. has reported significant improvements in quality of life outcomes of diabetics after pancreas transplant [19••]. When caring for individuals struggling with a very brittle form of diabetes, often in combination with subpar control of exocrine insufficiency, it becomes readily apparent that restoring normal physiology via pancreas transplant would go a long way toward improving health and social functioning.

\section{Mortality Risk}

Thirty-day perioperative mortality of TPIAT is $0-1.2 \%$, with overall long-term (5- and 10-year) survival of 90$94.6 \%$ and $81-84 \%$, respectively, with incremental im-

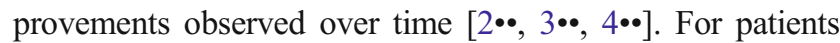
struggling with exocrine insufficiency and diabetes, there is a risk of mortality due to narcotic and carbohydrate absorption issues [20, 21•]. Five- and 10-year patient survival rates for PTA in Type 1 diabetics is 90.7 and $74.4 \%$, respectively [22•]. The existing literature on PTA for $3 \mathrm{c}$ diabetes is devoid of long-term data, but 3-year patient survival is $89 \%$ [10]. 


\section{Cost of Care}

While organ transplantation is expensive, it can reduce the cost of care of the organ failure patient. There is an emerging body of data regarding cost of care of patients with chronic pancreatitis. Wilson et al. compared the long-term cost of care for those undergoing TPIAT vs medical management only, finding $\$ 153,576 / 14.9$ quality adjusted life years (QALY) compared to $\$ 196,402 / 11.5$ QALY, respectively [4••]. In a cohort from the UK, Garcea et al. estimated the cost of undergoing TPIAT in the UK to be $£ 110,445$ [14••]. Multiple reports show a decrease in hospital admissions, length of stay, and opioid use, all of which decrease the cost of long-term expenditures in this population; however, the cost of care of

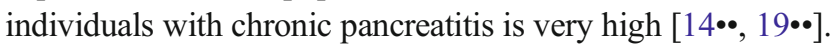
Even after a successful TPIAT (from a pain and endocrine stand point), the ongoing costs of exocrine replacement therapy are substantial, as the typical $\sim 90,000$ lipase units per day dosed by multiple tablets with every meal and snack costs between $\$ 24,000$ and $\$ 36,000$ per year [23 ${ }^{\bullet}$. Costs of replacing potential vitamin nutritional deficiencies (especially iron and vitamin D) are not yet quantified in the literature [24•]. For those patients experiencing brittle diabetes and/or malnutrition due to uncontrollable exocrine insufficiency requiring total parenteral nutrition (TPN), a good argument can be made for offering pancreas transplant $[25,26,27 \bullet \bullet]$. The first year cost of a pancreas transplant is about $\$ 125,000$ and then $\$ 7$ 20,000 annually for follow-up medical care $[28,29 \bullet \cdot]$. The cost of immunosuppression is estimated at $\$ 1000-3000$ per month (or $\$ 17,000-21,000$ per year) [23•, 30, 31•]. Taking on the medical and financial burden of chronic immunosuppression is not trivial and should be reserved for patients at high risk of progressive morbidity and mortality after failed IAT.

\section{Clinical Care of Patients Undergoing Pancreas Transplant After Pancreatectomy}

Our center's experience with PTA after TP or TPIAT has been previously reported and continues to grow [10-12]. Since
1986, we have performed 33 PTA in 25 patients, with overall 3 - and 5-year graft survival outcomes of 61 and $53 \%$, which is equal to those reported for a modern national cohort of PTA's transplanted in 2007 (Fig. 1) [29••]. In the current era (eight transplants done between 2005 and 2009), our 5-year patient survival rate is $100 \%$. This unique population of pancreas transplant recipients requires special attention to the nuances of their disease. Patient selection is similar to that of PTA for type $1 \mathrm{DM}$. While they have less duration of diabetes, they have other issues that impact morbidity and mortality after pancreas transplant. The intraoperative, perioperative, and posttransplant care of these recipients is slightly different than that of "traditional" Type 1 DM PTA patients.

As medication adherence is an underpinning of successful transplantation of any type, patient selection is paramount. Failure of an IAT or exocrine replacement therapy due to patient non-adherence predicts poor outcome after PTA and candidates should be screened well for these behaviors. The best candidates are those that have excellent adherence, uncontrolled metabolic issues, no significant residual pain syndrome, and good social support. These patients are more likely to be sensitized as they are more often female and more likely to have a history of blood transfusion (due to their complex surgical history and predisposition for chronic anemia due to nutritional issues). While our traditional approach was to perform bladder drainage to ensure better capture of rejection episodes by monitoring urinary amylase excretion and planning enteric conversion after the first posttransplant year, we have increasingly shifted our practice to choosing very well matched grafts and performing enteric drainage, often suture pexying the tail to the abdominal wall to ensure the ability to biopsy the graft. This approach affords immediate correction of exocrine deficiency, and may impact immunosuppression medication absorption. While there may be some physiologic advantage to portal venous drainage of a pancreas transplant, this population may not be well suited to this approach for several reasons [32-34]. As these patients may have a difficult surgical abdomen, it is important to avoid the selection of allografts predicted to have increased cold ischemic times. A good understanding of the patient's anatomy is important, and
Fig. 1 Graft survival rates for pancreas transplants done for surgical diabetes at the University of Minnesota (a) and for US pancreas transplant alone cases done in 2007 (b)
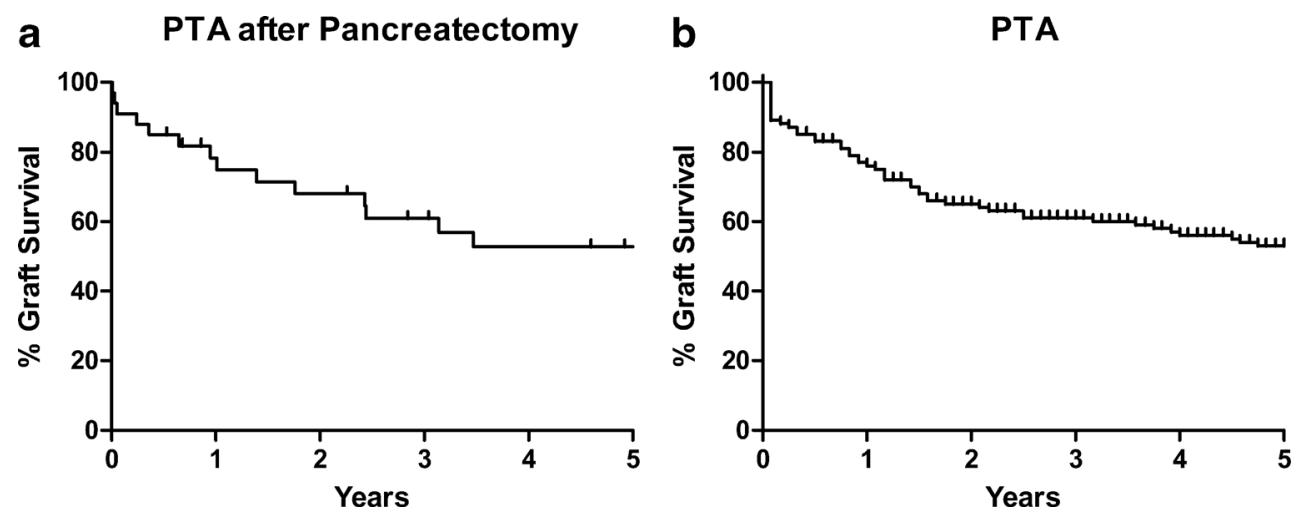
placement of the duodenoenterostomy at the proximal jejunum will allow for the most normal exocrine function. Consideration should be given for a surgical feeding jejunostomy, especially if the patient is TPN-dependent, is still on narcotics, or has significant nausea or gastroparesis, as motility disorders present a risk for reflux pancreatitis as well as rejection due to impaired absorption of immunosuppressive medications.

Splenectomy is often performed for technical reasons during TPIAT. The spleen is thought to be important in mounting a response to and clearing cytomegalovirus (CMV) infection [35]. Our clinical experience suggests that avoiding a CMV seropositive donor for a CMV seronegative recipient who is status post-splenectomy is helpful. The asplenic state is associated with a postoperative reactive leukocytosis and thrombocytosis that can confound interpretation of posttransplant laboratory studies and the patient's clinical course.

Expert perioperative pain management is important in this population, as they are sensitive to visceral pain and often have had traumatic experiences relating to prior hospitalizations and procedures. Avoiding the escalation of intravenous narcotic dosing in the postoperative period is important and can be achieved by addressing anxiety as well as utilizing adjunctive therapies such as the preoperative placement of paravertebral nerve blocks, intra- and postoperative use of dexmedetomadine, ketamine, gabapentin, ketorolac, and intravenous acetaminophen.

\section{Conclusion}

In the setting of surgical diabetes, whole organ pancreas transplantation treats two aspects of organ failure resulting from pancreatectomy - restoration of normal endocrine and exocrine function and thereby ameliorating the ongoing significant costs of care, morbidity, and risk of mortality in this subset of patients. While PTA results in this population are comparable to traditional PTA recipients over short-term follow up, questions remain about longer-term outcomes.

\section{Compliance with Ethics Guidelines}

Conflict of Interest Ty B. Dunn, Varvara A. Kirchner, K. Louise Berry, Melena D. Bellin, David E.R. Sutherland, and Timothy L. Pruett declare that they have no conflict of interest.

Human and Animal Rights and Informed Consent This article does not contain any studies with human or animal subjects performed by any of the authors.

\section{References}

Papers of particular interest, published recently, have been highlighted as:

- Of importance

• Of major importance

1.• Bellin MD, Gelrud A, Arreaza-Rubin G, Dunn TB, Humar A, Morgan KA, et al. Total pancreatectomy with islet autotransplantation. Summary of a National Institute of Diabetes and Digestive and Kidney Diseases Workshop. Pancreas. 2014;43(8):1163-71. Detailed summary of current knowledge, gaps, and unmet needs surrounding TPIAT (patient selection, indications, timing, technical aspects, outcomes).

2.• Sutherland DER, Radosevich DM, Bellin MD, Hering BJ, Beilman GJ, Dunn TB, et al. Total pancreatectomy and islet autotransplantation for chronic pancreatitis. J Am Coll Surg. 2012;214(4):409-24. discussion 424-6. Outcomes analysis from the Minnesota group for 409 TPIAT (adult and pediatric) done between 1977 and 2011, including mortality, islet cell transplant data, pain relief, endocrine and quality of life.

3.• Chinnakotla S, Radosevich DM, Dunn TB, Bellin MD, Freeman ML, Schwarzenberg SJ, et al. Long-term outcomes of total pancreatectomy and islet auto transplantation for hereditary/genetic pancreatitis. J Am Coll Surg. 2014;218(4):530-43. Comprehensive report of long term outcomes for TPIAT done for hereditary vs non-hereditary causes. Particular attention is given to the impact of timing of TPIAT and long term endocrine and pain outcomes.

4.• Wilson GC, Ahmad SA, Schauer DP, Eckman MH, Abbott DE. Cost-effectiveness of total pancreatectomy and islet cell autotransplantation for the treatment of minimal change chronic pancreatitis. J Gastrointest Surg. 2015;19(1):46-54. doi: 10.1007/ s11605-014-2612-3. The Cincinnatti group's experience with effectiveness of TPIAT vs medical management for minimal change chronic pancreatitis on decreasing hospital admissions, endoscopy and imaging as well as cost of care.

5. Bachmann K, Tomkoetter L, Erbes J, Hofmann B, Reeh M, Perez $\mathrm{D}$, et al. Beger and Frey procedures for treatment of chronic pancreatitis: comparison of outcomes at 16-year follow-up. J Am Coll Surg. 2014;219(2):208-16. A randomized controlled trial showing equivalent longterm outcomes after duodenal preserving Beger and Frey procedures.

6.• Crosby J, Bellin MD, Radosevich D, Chinnakotla S, Dunn TB, Pruett TL, et al. Gastrointestinal symptoms before and after total pancreatectomy with islet autotransplantation: the role of pancreatic enzyme dosing and adherence. Pancreas. 2015;44(3):453-8. New data from the Minnesota group on pancreatic exocrine replacement therapy after pancreatectomy.

7.• Ewald N, Bretzel RG. Diabetes mellitus secondary to pancreatic diseases (Type 3c) - are we neglecting an important disease? Eur J Intern Med Eur Fed Intern Med. 2013;24(3):203-6. An excellent review of Type $3 \mathrm{c}$ diabetes, including prevalence and clinical characteristics of endocrine and exocrine failure and alterations in gut physiology in this underappreciated and hence understudied population.

8. Slezak L, Andersen DK. Pancreatic resection: effects on glucose metabolism. World J Surg. 2001;25(4):452-60.

9. Hardt PD, Brendel MD, Kloer HU, Bretzel RG. Is pancreatic diabetes (type $3 \mathrm{c}$ diabetes) underdiagnosed and misdiagnosed? Diabetes Care. 2008;31 Suppl 2:S165-9.

10. Gruessner RWG, Sutherland DER, Drangstveit MB, Kandaswamy $\mathrm{R}$, Gruessner AC. Pancreas allotransplants in patients with a 
previous total pancreatectomy for chronic pancreatitis. J Am Coll Surg. 2008;206(3):458-65.

11. Gruessner RWG, Sutherland DER, Dunn DL, Najarian JS, Jie T, Hering BJ, et al. Transplant options for patients undergoing total pancreatectomy for chronic pancreatitis. J Am Coll Surg. 2004;198(4):559-67

discussion 568-9.

12. Gruessner RWG, Manivel C, Dunn DL, Sutherland DER. Pancreaticoduodenal transplantation with enteric drainage following native total pancreatectomy for chronic pancreatitis. Pancreas. 1991;6(4):479-88.

13. Gruessner AC, Sutherland DER. Pancreas transplant outcomes for United States (US) and non-US cases as reported to the United Network for Organ Sharing (UNOS) and the International Pancreas Transplant Registry (IPTR) as of May 2013. In: Cecka JM, Terasaki PI, editors. Clinical transplants 2003. Los Angeles: UCLA Immunogenetics Center; 2003. p. 21-51.

14.•- Garcea G, Pollard CA, Illouz S, Webb M, Metcalfe MS, Dennison AR. Patient satisfaction and cost-effectiveness following total pancreatectomy with islet cell transplantation for chronic pancreatitis. Pancreas. 2013;42(2):322-8. Outcomes data from the Leicester group comparing TP alone vs TPIAT, which shows that inclusion of the IAT is cost neutral, offers superior survival, and that TPIAT is cost effective when compared to nonsurgical therapy.

15. Alejandro R, Barton FB, Hering BJ, Wease S. 2008 update from the collaborative islet transplant registry. Transplantation. 2008;86(12): 1783-8.

16.• Bellin MD, Parazzoli S, Oseid E, Bogachus LD, Schuetz C, Patti $\mathrm{ME}$, et al. Defective glucagon secretion during hypoglycemia after intrahepatic but not nonhepatic islet autotransplantation. Am J Transplant. 2014;14(8):1880-6. The inclusion of a non-hepatic site for IAT showed preservation of glucagon secretion and symptom responses to hypoglycemia when compared to subjects with intrahepatic only IAT.

17.• Morgan K, Owczarski SM, Borckardt J, Madan A, Nishimura M, Adams DB. Pain control and quality of life after pancreatectomy with islet autotransplantation for chronic pancreatitis. J Gastrointest Surg. 2012;16(1):129-33. discussion 133-4. The MUSC group provides a detailed report of their experience with changes over the first year after TPIAT in quality of life scores and narcotic use.

18.• Walsh RM, Saavedra JRA, Lentz G, Guerron AD, Scheman J, Stevens T, et al. Improved quality of life following total pancreatectomy and auto-islet transplantation for chronic pancreatitis. J Gastrointest Surg. 2012;16(8):1469-77. The Cleveland Clinic's experience with pain, quality of life, and endorine outcomes in their prospective cohort (2007-2010). While endocrine outcomes were less that reported elsewhere, their group utilizes off site pancreas processing and therefore had longer cold ischemia times.

19.• Hall TC, Garcea G, Webb MA, Al-Leswas D, Metcalfe MS, Dennison AR. The socio-economic impact of chronic pancreatitis: a systematic review. J Eval Clin Pract. 2014;20(3):203-7. A review of the direct and indirect costs of care for patients with chronic pancreatitis in the United Kingdom, with sections on health care resource utilization, costs of endocrine, exocrine and pain management, as well as data specific to the cost and societal impact of chronic pancreatitis on health care systems in other countries.

20. Kahl S, Malfertheiner P. Exocrine and endocrine pancreatic insufficiency after pancreatic surgery. Best Pract Res Clin Gastroenterol Elsevier. 2004;18(5):947-55.

21. Jones CM, Mack KA, Paulozzi LJ. Pharmaceutical overdose deaths, United States, 2010. JAMA. 2013;309:657-9. An overview of the increasing trend of opioid associated overdose deaths in the United States.

22. 2011 Annual Report of the U.S. Organ Procurement and Transplantation Network and the Scientific Registry of Transplant Recipients. Department of Health and Human Services, Health Resources andServices Administration, Healthcare Systems Bureau Division of Transplantation, Rockville, MD; UnitedNetwork for Organ Sharing, Richmond, VA; Scientific Registry of Transplant Recipients, Minneapolis, MN. US data on pancreas transplantation activity, donor and recipient characteristics, graft and patient survival rates.

23. McCray S. Nutrition issues in gastroenterology. Pancreatic Enzyme Table Pract Gastroenterol. 2013;37(April):36. A detailed list of pancreatic enzyme therapy composition and cost and manufacturers.

24. Van Arsdale S, Goral S. Nutrition therapy for total pancreatectomy and autologous islet cell transplant. Support Line 2014;36(5):9-17. Nice overview of macro and micro-nutrient needs, and gastrointestinal issues that can impact management of patients who undergo TPIAT

25. Kiberd BA, Larson $\mathrm{T}$. Estimating the benefits of solitary pancreas transplantation in nonuremic patients with type 1 diabetes mellitus: a theoretical analysis. Transplantation. 2000;70(7):1121-7.

26. Demartines N, Schiesser M, Clavien P-A. An evidence-based analysis of simultaneous pancreas-kidney and pancreas transplantation alone. Am J Transplant. 2005;5(11):2688-97.

27.• Mehrabi A, Golriz M, Adili-Aghdam F, Hafezi M, Ashrafi M, Morath C, et al. Expanding the indications of pancreas transplantation alone. Pancreas. 2014;43(8):1190-3. The Heidelberg group's review of the world experience of pancreas transplant after pancreatectomy, outlining the observed improvements in quality of life and decrease in mortality. They hypothesize that pancreas transplant after pancreatectomy would be cost effective and not impact the organ supply in Germany.

28. Pancreas Transplant [Internet]. National Kidney Foundation. 2014. Available from: https://www.kidney.org/atoz/content/pancreastx.

29.• Israni AK, Skeans MA, Gustafson K, Schnitzler MA, Wainright JL, Carrico RJ, et al. OPTN/SRTR 2012 annual data report: pancreas. Am J Transplant. 2014;14(S1):45-68. The most current national US data on the cost and graft survival of pancreas transplant alone.

30. Immunosuppressive Drug Therapy [Internet]. 2014. Available from: http://medicareunder65.com/esrd-and-medicare/medicarefor-kidney-transplant-recipients/immunosuppressive-drugtherapy/.

31. Evans R. An actuarial perspective on the annual per patient maintenance immunosuppressive medication costs for transplant recipients 1993-2011. Am J Transplant. 2013;13(S5):270. Cost of immunosuppresion after organ transplant.

32. Rosenlof LK, Earnhardt RC, Pruett TL, Stevenson WC, Douglas MT, Cornett GC, et al. Pancreas transplantation: an inititial experience with systemic and portal drainage of pancreatic allografts. Ann Surg. 1992;215(6):586-95.

33. Reddy KS, Shokouh-Amiri H, Stratta RJ, Gaber AO. Successful reuse of portal-enteric technique in pancreas retransplantation. Transplantation. 2000;69(11):2443-5.

34. Stratta RJ, Alloway RR, Lo A, Hodge EE. Does surgical technique influence outcomes after simultaneous kidney-pancreas transplantation? Transplant Proc. 2004;36(4):1076-7.

35. Han XY, Hellerstedt BA, Koller CA. Postsplenectomy cytomegalovirus mononucleosis is a distinct clinicopathologic syndrome. American J of the Medical Sciences. 2010;339(4):395-9. 